\title{
Nonperiodic Octagonal Patterns from a Jali Screen in the Mausoleum of Muhammad Ghaus in Gwalior and Their Periodic Relatives
}

\author{
Emil Makovicky ${ }^{1}$ (D) Nicolette M. Makovicky ${ }^{2}$
}

Published online: 4 November 2016

(C) Kim Williams Books, Turin 2016

\begin{abstract}
One of the large jali screens adorning the mausoleum of Muhammad Ghaus in Gwalior (N India), built in 1565, contains panels composed of disordered composite octagons and Salomon stars. These elements show a rotational disorder with some interdependence. Analysis of these partially disordered patterns with rotatable configurations of the above elements suggested that they may be approximants of a quasiperiodic octagonal tiling based on a new type of composite tiles. Comparisons with the Amman's quasiperiodic tiling were made. Instances of similar or related periodic ornamental patterns at other northern Indian localities are analyzed as well.
\end{abstract}

Keywords Nonperiodic octagonal pattern · Jali screen · Dichroic octagonal quasiperiodic tiling · Muhammad Ghaus · Mausoleum · Gwalior (N. India)

\section{Introduction}

The times of The Great Mughals (1526-at least 1707) mark the blossoming period of the Indian Islamic architecture, culminating in the mausoleum of Taj Mahal (begun 1632). Large perforated screens, known as jali screens (Tillotson 1990; Broug 2013) through which light from outside filters to the interior are the most intriguing features of this architecture. They are carved from solid rock, marble or red Indian sandstone used in many important Indian buildings. The beautiful red

Emil Makovicky

emilm@ign.ku.dk

1 Department of Geoscience and Natural Resource Management, University of Copenhagen, Østervoldgade 10, 1350 Copenhagen K, Denmark

2 School of Interdisciplinary Area Studies, University of Oxford, 12 Bevington Road, Oxford OX2 6LH, UK 
sandstone and white marble imparted a specific character to the architecture of the region and enabled carving of the slender jali screens.

The mausoleum of a Sufi saint Muhammad Ghaus in Gwalior (Madhya Pradesh, N. India) was commissioned by the Mughal emperor Akbar. It was finished in 1565, at the beginnings of the Mughal architectural explosion (Takeo 2004; Porter and Degeorge 2009). It contains many large first-class stone-lattice jali screens. The best of them contains 20 smaller rectangular jali panels in the principal portion, six more in the tympanum and seven in partial screens above and beside them. It contains several non-periodic patterns which aroused our interest.

The majority of screen patterns are highly symmetric. In the rectangular screens, seven types of patterns are $p 4 \mathrm{~mm}$, one $p 6 \mathrm{~mm}$, and one frequently applied pattern pmm2. Related periodic patterns occur in several other localities, e.g., in the mausoleum of Hasan Khan in Sasaram, Bihar, India, built in 1535 by the alternative Suri dynasty (Porter and Degeorge 2009), in which the same choice of plane groups of symmetry is present. The carved and inlaid periodic patterns related to the nonperiodic panels treated in this paper are found at several north Indian localities of early-to-mature Mughal and post-Mughal eras, and will be dealt with briefly here.

\section{The Octagonal Patterns}

The most intriguing patterns which lack visual periodicity are situated in the lower corners of the bottom jali portion, and in the corners of the screen-like tympanum. The most prominent element in them is filled octagons of intermediate size, which appear to be chaotically distributed in the lower screens (Fig. 1).

These octagons are almost always overlapping with one or two adjacent octagons of the same kind, to the depth of $a / \sqrt{ } 2$, where $a$ is the length of the edge of octagon. The vast majority of them, the complete octagons, display three such overlap portions which, because of their shape, we call 'baguettes' (these are strongly compressed hexagons divided in two equal halves). Two of the baguettes are immediately adjacent to one another, and in contact, whereas the third one is poised symmetrically along the opposite edge of the octagon (Fig. 2). A small square below the center of the octagon, and a very open ' $\mathrm{V}$ ' of constricted, concave eightfold polygons (shaped as an incomplete number 8), complete the picture. The octagon point-group symmetry of $8 \mathrm{~mm}$ is reduced to $\mathrm{m}$.

A minority of unfinished/incomplete polygons has two symmetrically positioned 'baguettes' separated from one another. The third is situated asymmetrically 'above' them and partly outside. Often, the centre is filled by two constricted 8-gons in a ' $T$ ' configuration (Fig. 1). These incomplete octagons always overlap with other octagons (up to three overlaps) which are of the complete kind. In these unfinished octagons, one overlap destroys the integrity of the octagon boundaries. The interstices in the irregular agglomeration of octagons are filled by individual constricted 8-gons and adjacent small squares (Fig. 3a). 


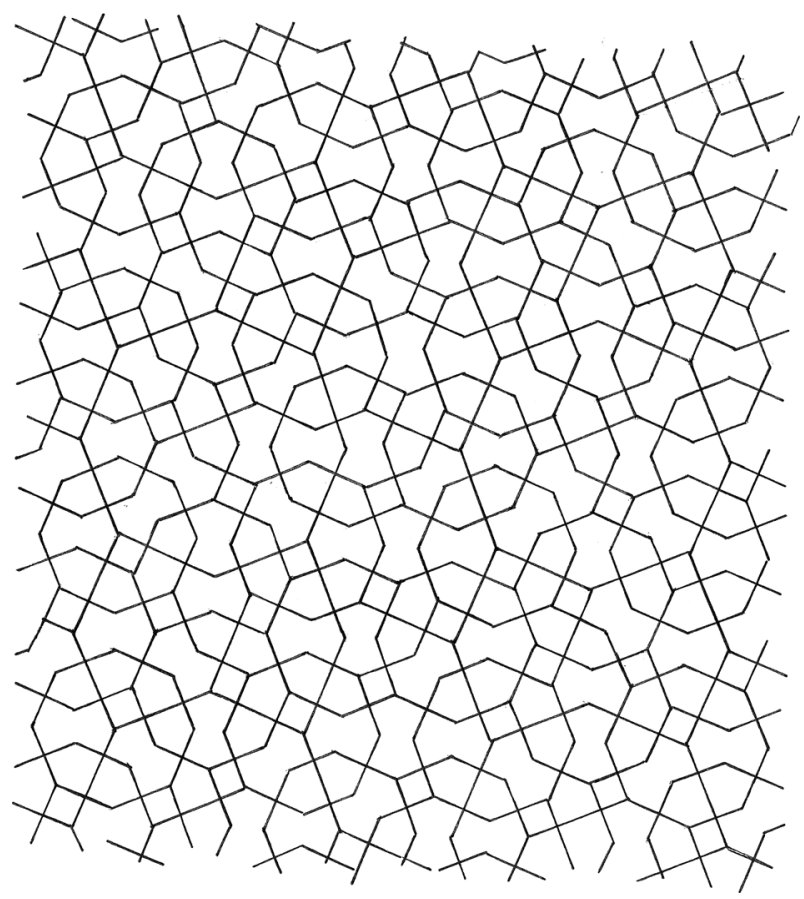

Fig. 1 A non-periodic panel from the large jali screen of the mausoleum of Muhammad Ghaus, a Sufi saint, in Gwalior, northern India, completed in 1565 under Akbar. Redrawn from a photograph
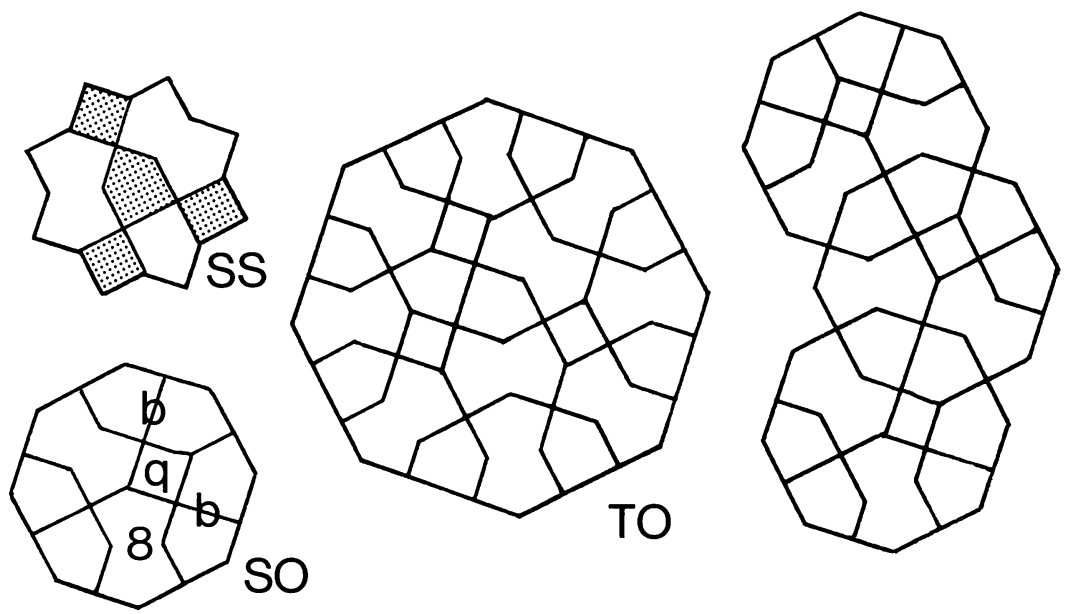

Fig. 2 Principal composite polygons from the Gwalior jali screen. A second-level alias secondary star (S-star, SS in the figure, the 'scorpion' configuration in it colored) and a composite second-level/ secondary octagon (S-octagon, SO in the figure) filled with primary $(P)$ tiles: compressed hexagons called here 'P-baguettes' (b in the figure), 8-shaped compressed/'pinched' P-octagons ( $\mathbf{8}$ in the figure) and small P-squares, denoted as q), center: a large third-level/tertiary T-octagon (TO) with halves of S-octagons distributed along the perimeter and, right: two of the attachment variations of S-octagons which partly overlap the central S-octagon via a common P-baguette 
a

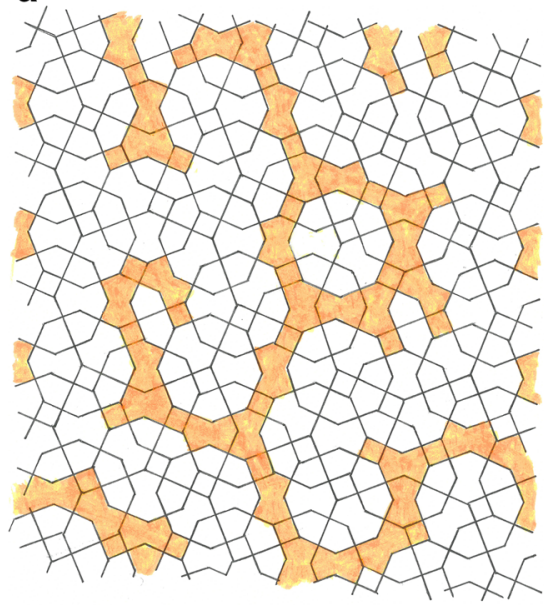

b

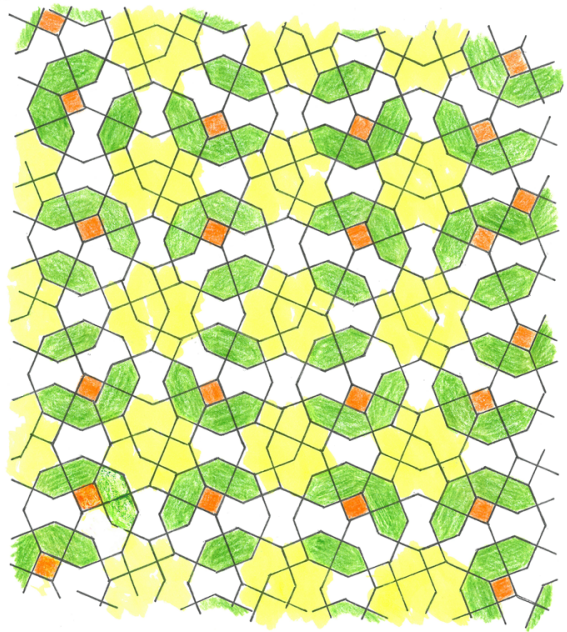

C

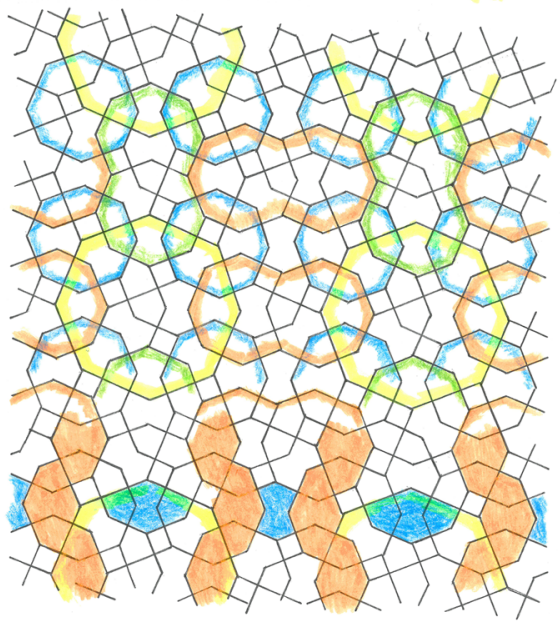

Fig. 3 a-c Interpretations of the non-periodic panel of composite S-octagons and S-stars from the bottom portions of the Gwalior jali screen, illustrated in Fig. 1. a Distribution of partly overlapping complete composite S-octagons (white) with interspaces (colored) composed of compressed 8-shaped P-octagons and small P-squares. b Lightly colored S-stars with a composite P-fill, and S-octagons which contain darkly colored 'P-baguettes'. Additional 8-shaped P-octagons (white) interconnect composite S-octagons. Comparison of $\mathbf{a}$ with $\mathbf{b}$ shows the inherent ambiguity of the pattern interpretation. $\mathbf{c}$ Above: a regular sublattice of complete (although rotationally disordered) S-octagons (dark-framed, square lattice) and pairs of partly overlapped S-octagons in two orientations in the interior of square 'subcells'. Light frames large T-octagons. Below vertical and horizontal rows of asymmetrical, lozenge-shaped and beveled $T$-hexagons forming together a rectangular net (color figure online)

\section{Fundamental and Derived Tiles}

In what follows, we describe three levels of tiling in the Gwalior patterns. Each higher level contains tiles of the previous stage. Where a confusion may arise, we denote the tiles as primary (the set of smallest tiles), i.e. P-tiles, secondary (the next 
level, S-tiles filled by P-tiles) and tertiary [the largest, T-tiles filled by (parts of) S-tiles]. The octagons described in the previous section, although conspicuous to the eye, represent secondary, composite S-tiles of the Gwalior pattern. The primary tiles are (a) the compressed, flattened (and halved) hexagons, i.e., the above mentioned ' $(P$-)baguettes', (b) the hour-glass-like compressed concave P-octagons (shaped as Fig. 8) which combine with the angular ends of the 'P-baguettes', and (c) the small $P$-squares with the edge length equal to a half of the straight edge of the P-baguette and also to the concave edge of the small 8-shaped P-octagon. The small P-square (dimensions $a / 2 \times a / 2$ ) is a fundamental measure unit of the pattern: it defines one half of the long edge of the P-baguette (i.e., $a / 2$ as defined above) which equals length of the beveling edge of the baguette and equals any edge of concave P-octagon. Thickness of the P-baguette (extent of the overlap of two S-octagons) is a diagonal of the $\mathrm{P}$-square and the inscribed radius of the $\mathrm{S}$-octagon is equal to twice the edge plus twice the diagonal of the P-square (Figs. 1, 2, 3).

The overlapping incomplete S-octagons and the small filling P-elements generate another secondary element: a filled eightfold S-star (with $90^{\circ}$ teeth, traditional artisan name 'Salomon star') (Figs. 2, 3b). It contains one P-baguette, two symmetrically disposed concave P-octagons and three small P-squares situated at three of its vertices. Therefore, the secondary-level pattern consists of (a) perfect secondary S-octagons in a well-defined partial overlap, (b) secondary $S$-stars and, (c) lone concave P-octagons which act as interconnections of the elements sub (a) and (b).

The still larger tertiary octagons do not overlap (Figs. 2, 3c). They are separated from each neighbor by $S$-stars and they contain an $S$-star in the center, surrounded by a wreath of concave P-octagons which point to the vertices of the large T-octagon in which they reside, and are interspaced by halves of P-baguettes which are centered on the edges of the large octagon.

Do we know any analogies? Perhaps: Makovicky and Fenoll (2001) (quoted as M \& F tiling below) constructed a dual of the square tiles of the octagonal aperiodic tiling, with lines bending smoothly through the tips of lozenge tiles (Fig. 4). These lines outline S-octagons similar to the just described ones: two overlaps via 'Pbaguettes' correspond to the overlaps observed in the present case but the 'Pbaguettes' are separated from one another in the M \& F case. The third overlap in Gwalior tiling is symmetrically opposed to the two overlaps and does not exist in the M \& F tiling which has an interconnecting rhomb instead (Figs. 2, 4). The ' $\mathrm{V}$ shaped' analogy of two adjoint P-octagons in the Gwalior pattern is present in the $\mathrm{M}$ \& F pattern, although the small eccentric P-square of the Gwalior pattern is reduced to a central point. The 'uninterrupted line marking' of M \& F (2001) is based on a background of quasiperiodic tiling of squares and lozenges but the Gwalior pattern cannot be redrawn using only these two components.

It is the T-octagons which suggest kinship between the two tilings: in both tilings they have a central S-star, and concave S-octagons and half-baguettes organized along the perimeter-this means a wreath of eight S-octagons positioned on the vertices. In the Gwalior patterns, the T-octagons have a diameter $2 a+2 a / \sqrt{ } 2$ and are positioned on nodes of a large-scale square lattice (Fig. 3c). As mentioned they host eight S-octagons, positioned in the vertices; one or two of them may be of the 


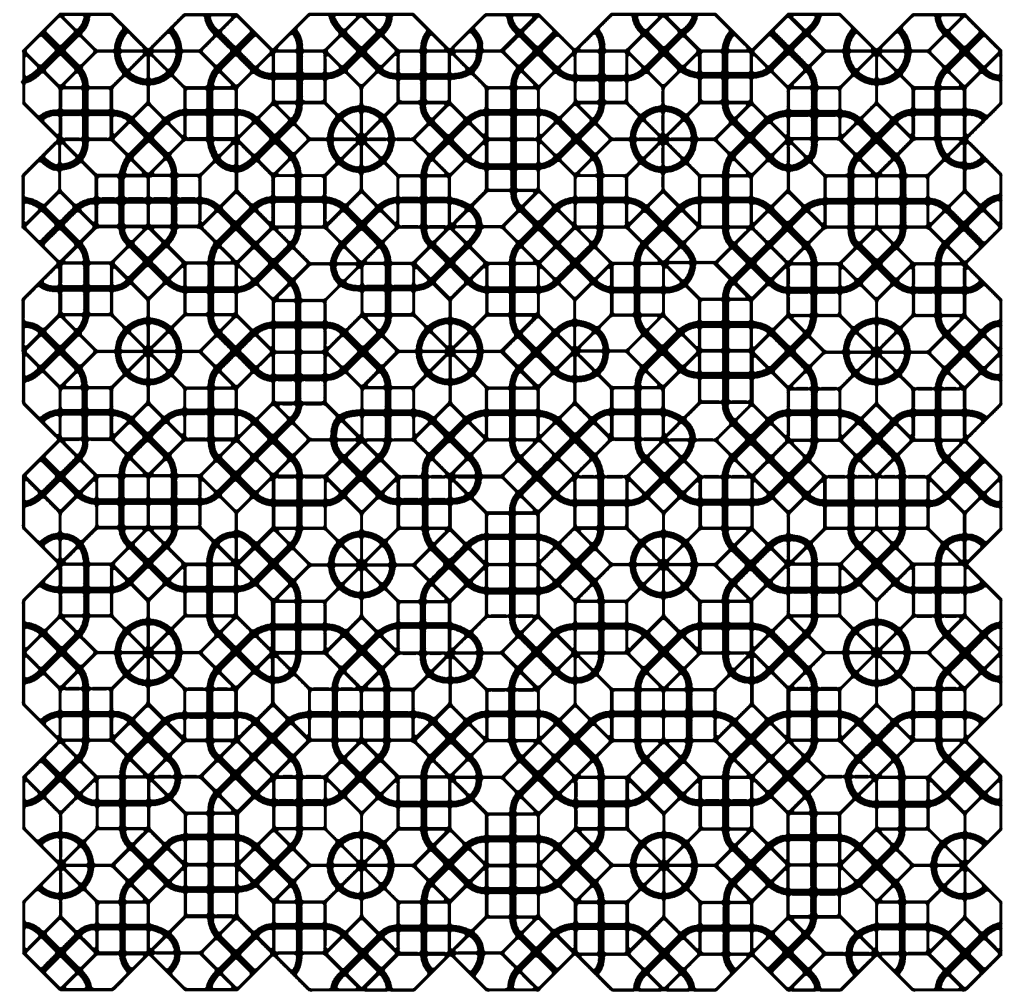

Fig. 4 A dual of the quasiperiodic octagonal pattern of Ammann squares and $45^{\circ}$ lozenges (background) derived by Makovicky and Fenoll (2001)

'unfinished' type (see above), just as in the M \& F tiling. At Gwalior, the eightfold $S$-star in centers of these large octagons contains a small 'scorpion design', which is composed of an irregular P-pentagon (half of the baguette) and three small P-squares (Fig. 2). The 'scorpion' is flanked symmetrically by two compressed P-octagons; these are the above mentioned 'figures of eight'; the symmetry of this arrangement is $m$. These large T-octagons must not be confused with the above analyzed S-octagons. In small panels of white-and-red marble on the walls of Akbar Sikandra mausoleum (completed in 1613 under Emperor Jahangir) the S-stars of this superoctagon pattern are adorned by a red, smaller star, raising the local symmetry to $8 \mathrm{~mm}$ though a continuation of the pattern is cut off (Porter and Degeorge 2009).

Partial overlap of one complete S-octagon and one unfinished S-octagon with the internal T-like configuration of P-octagons (see above) also leads to an $S$-star which straddles their line of overlap, and has an asymmetrically oriented 'scorpion' inside, analogous to the configuration in the T-octagon (Fig. 3b). The orientation of the scorpion group is dictated by the orientation of the complete S-octagon in the pair. This creates orientational ambiguity in the secondary pattern, and is its fundamental property. 
In the entire pattern, the overlapping S-octagons form zig-zag chains and chain fragments, occasionally interconnected laterally by an additional S-octagon (Fig. 3a). There is also a periodic 'skeleton' present, i.e., a square lattice of $\mathrm{S}$ octagons although with variable orientation of their fill (Fig. 3b, c). Those $\mathrm{S}$-octagons which are not on the nodes of this lattice are paired, either as rows of vertical pairs (with a doubled repetition period) or of horizontal pairs (every period) (Fig. 3c). The perfect S-octagon participating in such pairs of octagons is selected at random. All pairs create $S$-stars in the overlap or between two T-octagons (Fig. 3b). A wreath of overlapping S-octagons positioned at all corners of T-octagons is always present in the pattern. Such patterns are developed as random patterns in the larger principal pattern fields at the bottom of the jali (Fig. 1).

Interestingly, the M\&F pattern exhibits a similar 'lattice' of regularly spaced S-octagons with a randomly oriented fill, and with random P-elements situated between them, but they are rotated by $22.5^{\circ}$ relative to those in Gwalior and differently spaced (Fig. 4).

\section{Why is the Pattern Random, and to What Degree is it Random?}

The connecting lines radiating from the interior of the composite S-octagon are perpendicular to, and situated in, the midpoint of every S-octagon edge (the small P-squares attach themselves to these lines from the outside) (Fig. 2). Therefore, the S-octagons can rotate by $n \times 45^{\circ}$ without disturbing the coherence of the line pattern. Then we attach the next, partly overlapping S-octagon. For executing the overlap with the original S-octagon we have a free choice: either to use one of the two twin-joined 'P-baguettes' or the 'lone' P-baguette in the newly attached $\mathrm{S}$-octagon. This results in three possible orientations of the new S-octagon (Fig. 2, right-hand part).

As already mentioned, the concentric arrangement of half-baguettes and 'eightshaped' P-octagons along the perimeter of the T-octagon creates an eightfold S-star in its center. The small 'scorpion' in this S-star can rotate freely, by increments of $45^{\circ}$, without destroying the connectivity of the line pattern (Fig. 2), because connectivity of the S-star involves only its perimeter, and not the lines from its interior. In this way the pattern is similar to the decagonal aperiodic Maragha pattern from Iran, which contains (in our notation) rotatable S-pentagons and tenfold S-stars, both with a complicated P-fill which defines only a mirror symmetry (Makovicky 1992, 2008). These rotatable S-pentagons and decagonal S-stars correspond in principle to the present, filled S-octagons and part of the S-stars.

In some jali patterns of the Ghaus mausoleum, and in those from the Divan e-Khas, Lahore (1637) (Porter and Degeorge 2009), with a $\mathrm{cmm}$-based background configuration, the 'scorpions'and overlapping portions of paired S-octagons (with the concealed S-stars) were replaced/filled by 'open-arm swastikas'. The periodically-to-nonperiodically arranged partial rotations and orientations of these swastikas are analogous to the variations observed in the scorpion patterns just described. In a window of Humayun's Tomb (1557-1565, built by Mirak Mirza Ghiyas) in Delhi, which is a twin configuration with overall symmetry $2 \mathrm{~mm}$, only 
Fig. 5 A panel from the tomb of Humayun in Delhi, built between 1565 and 1572, with parallel-oriented dumb-bells of S-octagons and open-arm swastikas. It consists of four blocks, each with (approximate) oblique symmetry; the resulting twin (fourling) has $2 \mathrm{~mm}$ pointgroup symmetry

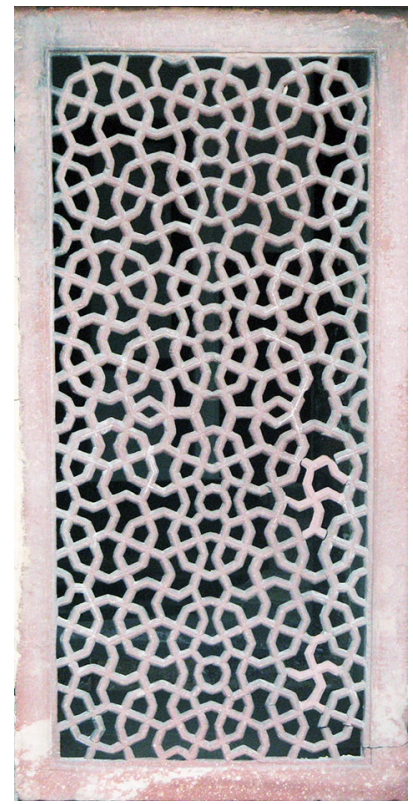

vertically oriented octagon pairs exist, in an overall $\mathrm{cmm}$ arrangement. The 'openarm swastikas', however, double the vertical periodicity by their alternating orientations (Fig. 5). Composition planes of the twin divide the pattern into four blocks. The symmetrized S-octagon pairs seen on the composition planes were exclusively used in a jali from Humayun's Tomb, illustrated in Fig. 6. It is a saturated $\mathrm{cmm}$ pattern for which the architect chose a 'quiet' horizontal orientation of $2 \mathrm{~mm}$ overlapping-octagon pairs.

\section{What is the Underlying Scheme of the Gwalior Pattern?}

As already mentioned, at the bottom portions of the Gwalior jali, the large T-octagons with their wreath of complete S-octagons are placed on a square lattice and a part of S-octagons form a square lattice of their own, with a halved periodicity (when we disregard the orientation of their P-fill) (Fig. 3b, c). Thus, the random character of the pattern (Fig. 3a) is masking a periodic lattice which serves as a basis of the entire pattern and details of the pattern allow more than one interpretation.

The distances between the T-octagons are determined by dumb-bells formed by two partly overlapping S-octagons which are situated on the vertices of two adjacent T-octagons (Fig. 3c). As a quite different description, the jali pattern contains a rectangular grid of horizontal and vertical lines of asymmetrical compressed (i.e., lozenge-shaped) composite S-hexagons, each composed of halves of two different P-baguettes and an intermediate constricted '8-shaped' P-octagon (Fig. 3c, lower portion). The vertical lines accommodate these $S$-lozenges in a glide-plane-like 
Fig. 6 A high-symmetry, cmmversion of the dumb-bell pattern. A jali screen from the tomb of Humayun in Delhi

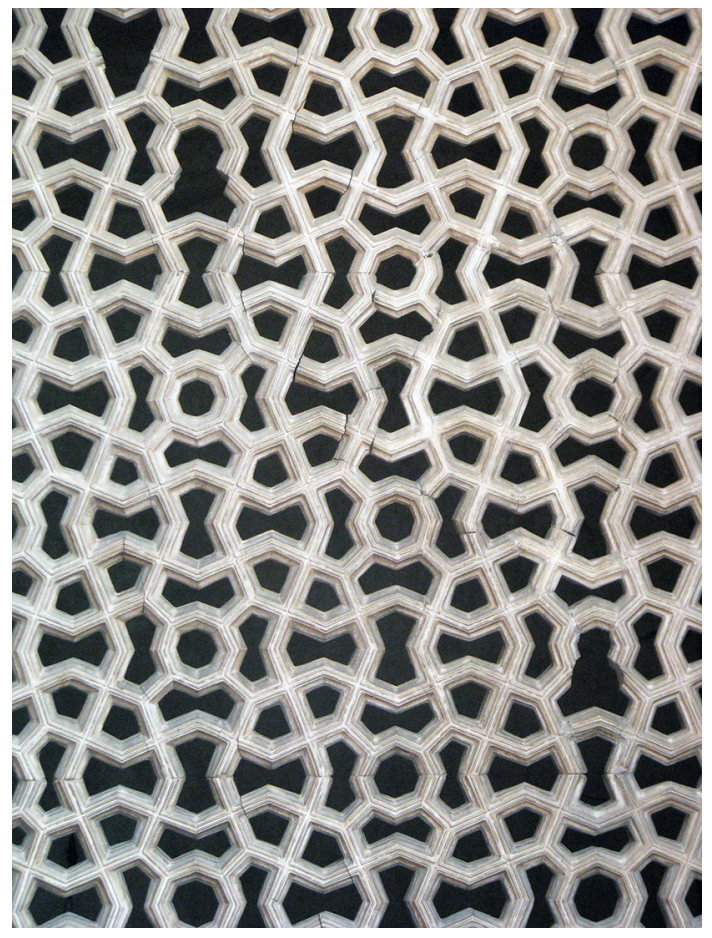

manner (both colored in Fig. 3c). Lozenge orientations in adjacent vertical lines are opposite. Orientation of the 'scorpions' in the S-stars is independent of the orientation of these lozenges; they can even all be parallel-oriented. When the orientation of the fill of the S-octagons is disregarded, the periodicity of the square pattern equals that of emptied S-stars (Fig. 3b). This was utilized in the frieze pattern from the Jama Masjid of Fatehpur Sikri, built from 1571 to 1575 in Agra, Uttar Pradesh. This frieze (Fig. 7) is a cut-out of a $22 \mathrm{~mm}$ pattern and shows several intriguing analogies to Fig. $3 \mathrm{~b}$.

\section{What is the Nature and Symmetry of this Random Pattern?}

As already mentioned, the T-octagons in the nodes of the just described tetragonal lattice are adorned with radially oriented P-baguettes straddling their edges, and with the constricted P-octagons below each of their vertices. This arrangement produces eight partly overlapping S-octagons decorating the T-octagon, four of which are engaged in overlapping dumb-bells of S-octagons. There is a forced pair of dumb-bells of overlapping S-octagons with the 'right-left' orientation, and also such a pair oriented perpendicular to the first one, with a vertical orientation (Fig. 3c).

The orientation of the remaining four S-octagons around a T-octagon, those which participate in a square lattice, is fixed by having two of their three P-baguettes 
Fig. 7 A frieze from Jama Masjid of Fatehpur Sikri, Agra, a cut-out of a $p 2 \mathrm{~mm}$ pattern of red sandstone and white marble with selected elements accentuated in black

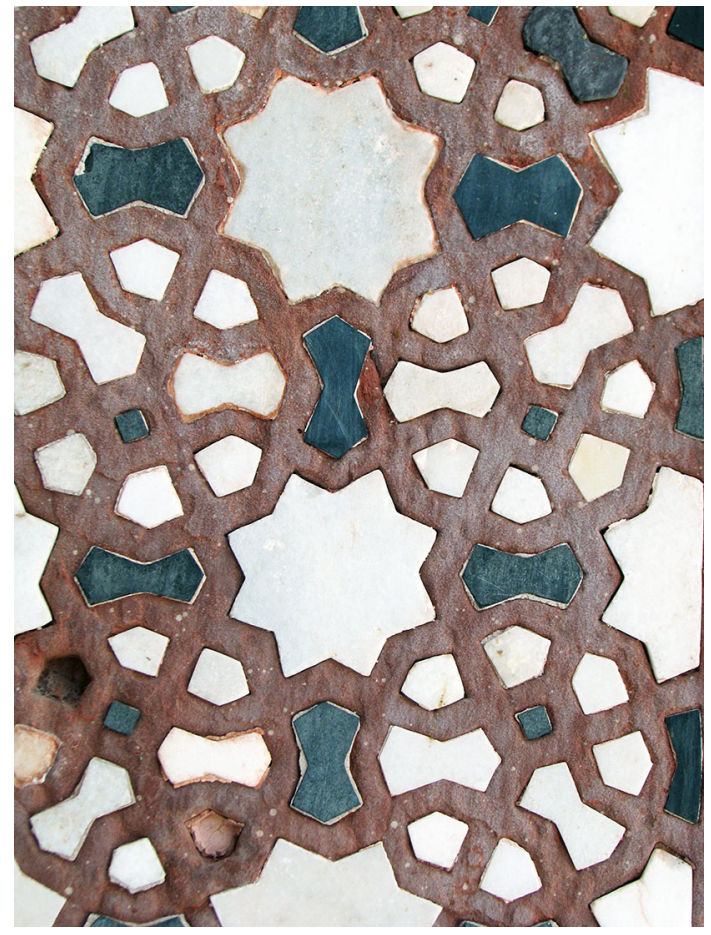

serving as the radii of the T-octagon on which they are placed. This leaves for them a double choice for the orientation of the third P-baguette and of their entire P-fill. As seen in Fig. 3b, the master artisan used this orientational freedom and arranged them into a pmm scheme with a periodicity equal to that of T-octagons (barring some mistakes).

To summarize our observations, when the fundamental P-tiles are ignored, the general underlying symmetry of this design is $\mathrm{pmm}$ with a periodicity one half of that of T-octagons. The filled S-stars (discs with symmetry $m$ ) rotate freely in the interior of the T-octagons, isolated from the rest by the high $8 \mathrm{~mm}$ symmetry of the perimeter. There is a free twofold random choice for the orientation of each complete S-octagon which participates in the boundary of the T-octagon (but there is a fixed orientation of the T-like tip-to-waist contact of two P-octagons inside a defect S-octagon), and then a threefold choice for the attached S-octagons needed to complete the zig-zag chains of S-octagons. The selection, which one of the two S-octagons of each dumb-bell pair is an incomplete octagon (only one of them can be 'complete' or both are incomplete, always violating the pmm symmetry), still leaves ambiguity in the orientation of the resulting 'scorpion' created inside the dumb-bell. There is no correlation of the 'complete S-octagon-incomplete S-octagon' orientations between adjacent dumb-bell-like pairs (Fig. 3c). All this produces the randomized pattern of the Gwalior jali screen, in which the underlying periodicity visually all but disappears. 
Thus, the Gwalior patterns are heavily randomized patterns based on a periodic distribution of certain motif elements with a fixed position but with a randomized element-fill orientation, and with a similarly constructed and randomized matrix between them, in which random element orientation generates a form of positional disorder. Recently, Castéra (2016) illustrated examples of 'rotationally-disordered', originally periodic patterns based on a decagonal Kond tile set in Isfahan.

\section{Quasiperiodic Character?}

From our experience with the decagonal quasiperiodic patterns and the related periodic patterns from Maragha (Iran) (Makovicky 1992, 2009, 2015) and Alhambra (Spain) (Makovicky et al. 1998), the presence of freely rotatable composite tiles suggests that the tile-set of the pattern might be (at least closely related to) a set of tiles with a potentially quasiperiodic character. Our investigation of the quasicrystalline capability of the Gwalior tile set concentrates upon the intermediate-level composite tiles', i.e., the $S$-octagons and $S$-stars. One approach used by us is to start from the above described T-octagon with a wreath of S-octagons and continue constructing a radial nonperiodic pattern from this nucleus. The other approach is to take a large-scale scheme of octagonal quasiperiodic tiling composed of squares and $45^{\circ}$-lozenges, designed by Ammann (Grünbaum and Shephard 1987; Makovicky and Fenoll 2001), and decorate its nodes in an organized way with Gwalior-type octagons and Salomon stars in mutual contact.

While the concept of one- or two-dimensional periodicity and non-periodicity is intuitively clear, we interpret quasiperiodicity as a case of non-periodicity guaranteed by special tile-edge and tile vertex markings and appropriate tilecomposition rules, resulting in an underlying two-dimensional quasiperiodic lattice as defined by Ammann (Grünbaum and Shephard 1987). Unmarked historic tilings can be considered 'practically' quasiperiodic when a sufficiently large tiling patch coincides in full with an equally representative tile patch of a guaranteed quasiperiodic tiling (with a sufficient number of spacings and spacing pairs of the Ammann quasilattice). Approximants are periodic patterns which are based on the tile types and preferably also tile combinations originating from the quasiperiodic patterns.

In the first of the two approaches (Fig. 8), after a wreath of partly overlapping $\mathrm{S}$-octagons, a ring of S-stars spaced by single concave P-octagons is generated, with a ring of S-octagons surrounding them. Then, a multiplicity of element stackings becomes possible. In the interpretation of this radial non-periodic pattern as Ammann's tiling, the Ammann lozenges originating from the center of the T-octagon have S-stars at the acute vertices and a pair of partly overlapping S-octagons at the obtuse vertices. The adjacent Ammann squares have two $\mathrm{S}$-octagons and two S-stars in a trans-configuration but the lozenges which follow after them have distribution of S-octagons and S-stars at their vertices opposite to that in the first set of lozenges. These two types of lozenges will then occur throughout the extended pattern. Elliptical aggregations of ten overlapping S-octagons result in a parallel accretion of another S-star to the existing S-star in 


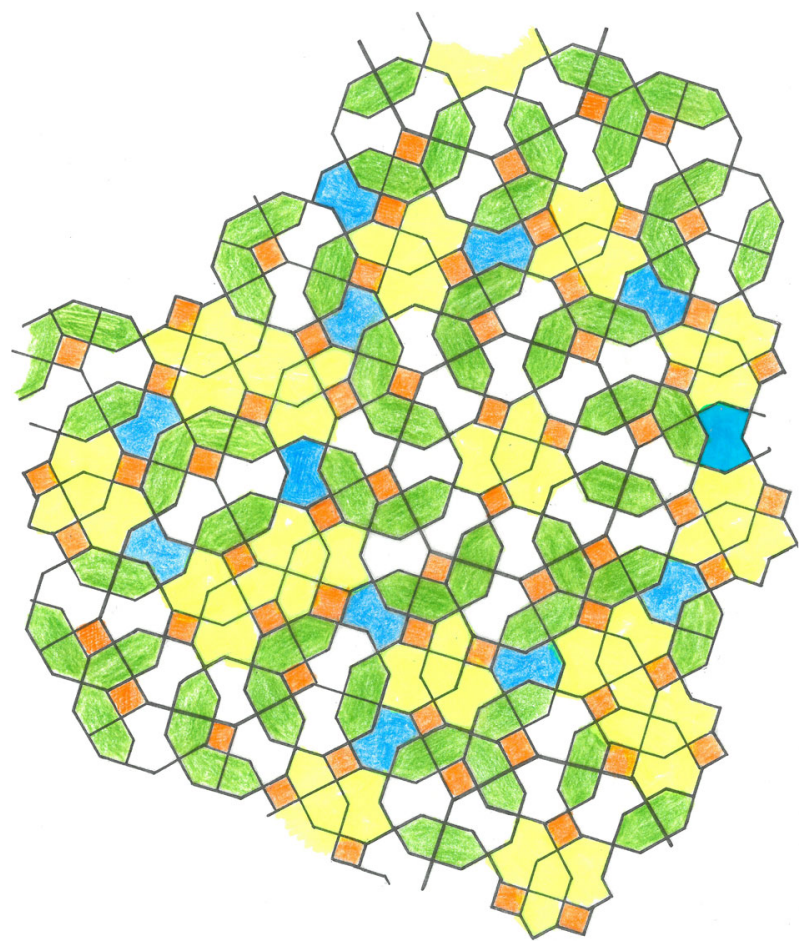

Fig. 8 Aperiodic collage of S-octagons (their fundamental P-tiles are colored) and S-stars (yellow, including the orange squares) of the Gwalior type. The collage starts from a composite T-octagon (stronger outline) in the pattern center, with a wreath of overlapping S-octagons along the perimeter. 'Surplus' 8-shaped interconnecting P-elements are blue (color figure online)

the center of the aggregation. The two types of Ammann lozenges mentioned are perpendicular to one another and partly overlapping in this configuration, i.e., there is ambiguity of interpretation. Our worry concerning a possible mixture of the allowed and forbidden tile adjacencies in this complex pattern was rather unexpectedly solved. When the S-octagons and S-stars situated in the vertices of Ammann tiles are, respectively replaced by white and black discs, our pattern is identical with a dichroically colored standard octagonal quasiperiodic tiling with a rather complex dichroic superspace group symbol $P_{a^{\prime}} 8 \mathrm{~mm}\left(8^{3} \mathrm{~mm}\right.$ ) (Janssen et al. 2007). Instead of rather voluminous explanations on superspace and analysis of the symbol, we illustrate this dichroic superspace group graphically and understandably for everybody in Fig. 9.

The second approach, in which we decorate vertices of the Ammann square-andlozenge scheme, uses a non-cartwheel drawing of the octagonal quasiperiodic pattern published by Grünbaum and Shephard (1987). S-octagons were put at the acute vertices of the star-like lozenge cluster, and S-stars at the obtuse vertices (Fig. 10a, b). Ammann squares have a trans-configuration scheme of S-octagons and S-stars. An incomplete wreath of S-stars generated from an incomplete star-like cluster of Ammann's lozenges is surrounded by a circle of S-octagons 


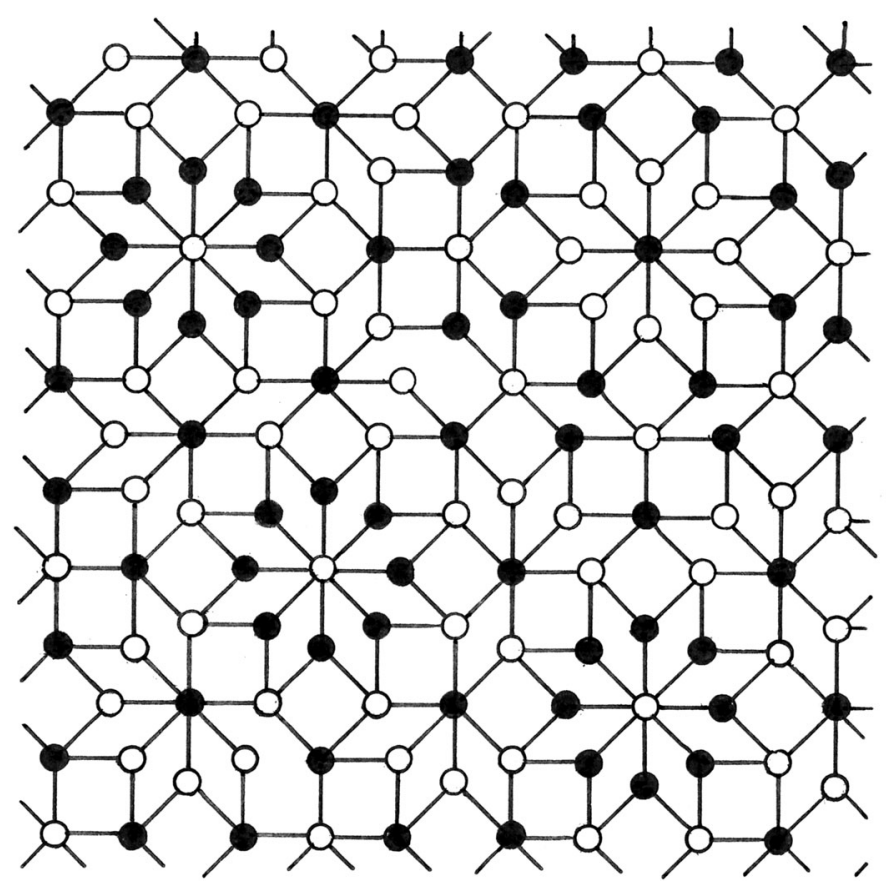

Fig. 9 A dichroically colored octagonal quasiperiodic pattern of Ammann lozenges and squares with a dichroic quasiperiodic superspace group $P_{a^{\prime}} 8 \mathrm{~mm}\left(8^{3} \mathrm{~mm}\right.$ ) (adapted from Janssen et al. 2007 with alterations)

interconnected by small concave P-octagons and by a variety of configurations afterwards. These include clusters of lozenges which generate star-centered rings of partly overlapping S-octagons (Fig. 10a).

The described distribution of S-octagons and S-stars over the Ammann scheme does not reproduce Ammann's aperiodicity markings in any way although it generates the dichroic character of the tiling. An analogous scheme, however, is the most straightforward interpretation of the M \& F pattern, mentioned previously, as well. The nature of the scheme is revealed by the fact that, because of the already mentioned ambiguities of interpretation, the primary star-and-octagon distribution over the vertices of Ammann's squares and lozenges generates a number of potential S-star locations attached in parallel to, and partly overlapping with, the existing S-stars which were determined by the Ammann tiling (Fig. 9a). These sites can be occupied by S-stars instead of the original sites which they partly overlap. On the level of Ammann tiles this ambiguity corresponds to a tile flipping, the simplest of which is a flipping of a group of two lozenges plus one Ammann square by $180^{\circ}$, resulting in an alternative position of the Ammann square (try to perform it in Fig. 9b). Thus, the pattern we constructed in Fig. 10a includes nodes of both flipping orientations. The original one is indicated by saturated coloring whereas the alternatives by light coloring. This recalls the ornamented lozenge-shaped P-tile in the decagonal Maragha tiling (Makovicky 1992, 2009), which simultaneously 

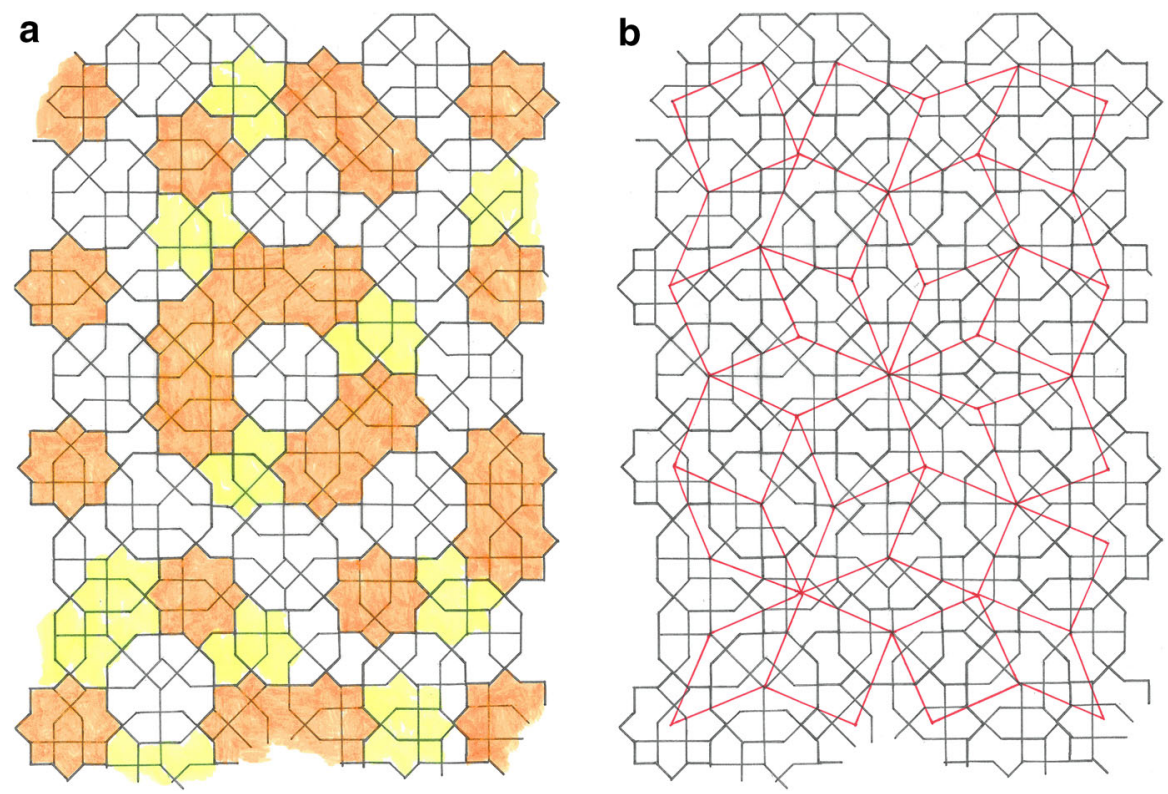

Fig. 10 a Aperiodic collage of composite S-octagons (uncolored) and S-stars (intensely colored), obtained by decorating vertices of Ammann's octagonal quasiperiodic tiling of squares and lozenges (shown as a red tiling in $\mathbf{b}$ ) by the octagons and stars which are in direct contact. Potential $S$-star positions occupied by flipping one of the adjacent stars from its current site are light-colored. Note S-octagoncentered rings of amalgamated stars, and $S$-star centered (incomplete) rings of overlapping octagons. Compare Fig. 10b with Fig. 9: S-octagons and S-stars stand for the two colors of the dichroic pattern (color figure online)

defines, through its shape and orientation, both partly overlapping positions of a freely flipping pentagon. The original Penrose tiling contains only one of these alternative positions at a time.

Thus, similar to the Maragha pattern, the Gwalior-type pattern can accommodate all possible variants/orientations of the Ammann tiling, and not simply one variant. Similar to the Maragha pattern with its rotatable filled S-pentagons and rotatable filled tenfold S-rosettes, it has two rotating composite elements- the filled S-stars and the filled S-octagons. These two 'interact' and must be correlated in the case of overlapping S-octagons or incomplete S-octagons (as defined above) recalling a similar situation in a decagonal tiling derived by Makovicky et al. (Figure 16a, 1998) for the decagonal tiling from the Alhambra.

To summarize, we demonstrated that both the Gwalior-type tiling and the Makovicky and Fenoll tiling are compatible with the octagonal quasiperiodic tiling. They have all its orientational varieties already potentially incorporated but they lack Ammann's quasiperiodicity markings. The presence of two differently decorated lozenge types in each of these tilings demonstrates the dichroic character of our Ammann tiling. Attempts to draw another Ammann tiling from the Gwaliorbased pattern, which would not have dually decorated lozenges, meet with problems outside the tiling nucleus. 


\section{Origins and Next of Kin}

From where comes the very special S-octagon of the Gwalior patterns? The answer is hidden in the jali screen at Lahore Fort (built after 1605, under Jahangir) (Stierlin 2002). It contains larger, and stronger-outlined, T-octagons, that in all details correspond to those at Gwalior (Fig. 11). However, at Lahore they share common edges, resulting in a classical pattern of T-octagons and T-squares, defined as $8^{2} .4$. The eightfold $S$-stars hosting (sometimes randomly oriented) 'scorpions', exactly as in the Gwalior pattern, are in the centers of T-octagons (Figs. 2, 10). The array of $\mathrm{S}$-octagons occupies all vertices of T-octagons, and together they outline the just mentioned S-stars.

As at Gwalior, the S-octagons in this pattern contain 3 P-baguettes (two of which are in contact), an open $\mathrm{V}$ configuration of constricted P-octagons, and a small offcenter P-square. This pattern explains the origins of the open $\mathrm{V}$ configuration: for any S-octagon centered on the apex of the T-octagon, the two arms of the $\mathrm{V}$ formation (which straddle the edge of the large T-octagon) point exactly to the centers of those adjacent T-octagons which share this S-octagon. Eight of these arms (the smallest, concave P-octagons), interspersed by half-baguettes, form the rotationally symmetric outer wreath of each T-octagon (Fig. 11). The S-stars in the $\mathrm{T}$-octagon centers can rotate freely, assuming eight orientations, but the S-octagons have orientations fixed in this pattern. Their small P-squares are in the interior of poised 'square T-tiles' of the large-scale $4.8^{2}$ pattern, and they outline a small but conspicuous cross of halved P-baguettes (Fig. 10). In the vertical direction, the constricted P-octagons form double rows, with rows of aligned 'P-baguettes' interspersed with small P-squares in their median lines. The resulting symmetry varies between the plane group $\mathrm{pm}$ and a random pattern.

The same $4.8^{2}$ pattern has been used for a jali at Chandra Mahal, within the City Palace, Jaipur, which was built between 1729 and 1732 (Fig. 12). Here, however, the diagonally oriented 'P-bagettes' are thicker than the horizontal and vertical set,

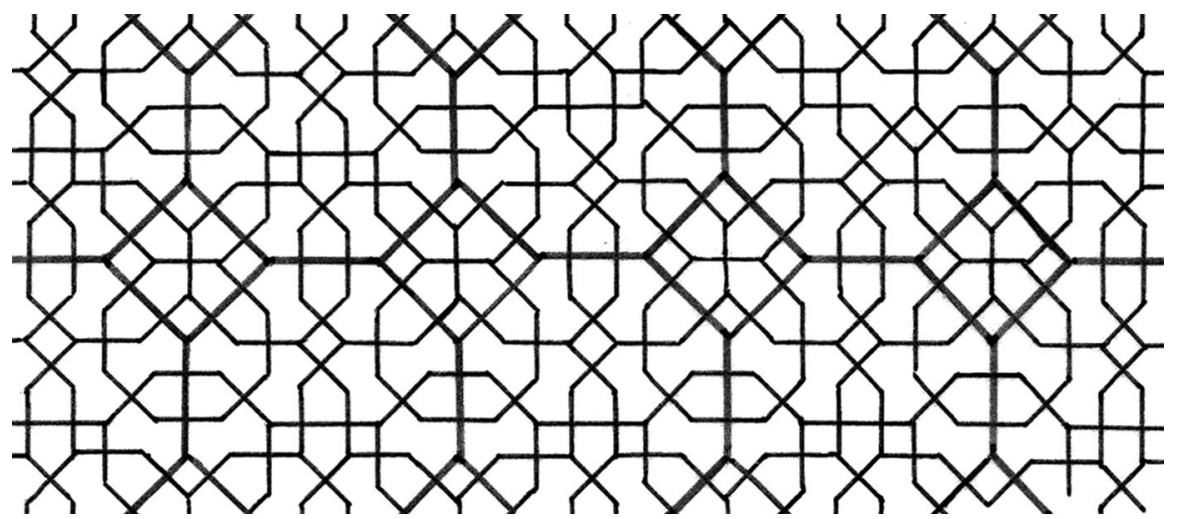

Fig. 11 A jali from the Lahore Fort, constructed under Jahangir (after 1605), a fully worked out $4.8^{2}$ pattern of T-octagons in red sandstone 
Fig. 12 A jali from Chandra Mahal, within the City Palace, Jaipur, built in 1729-1732. It is a late and partly modified version of the openwork screen shown in Fig. 10

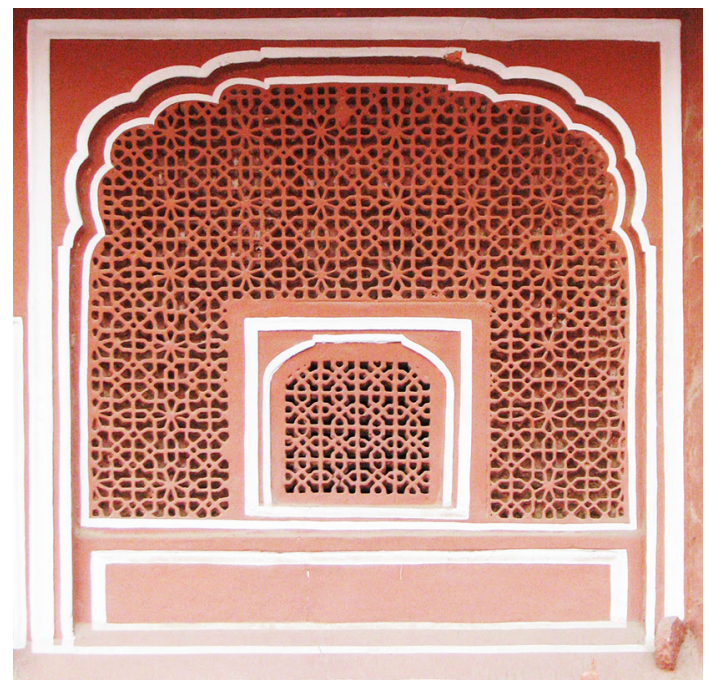

and the S-star has been filled by a rosette instead of the $m$-symmetric 'scorpion', preserving the $p 4 \mathrm{~mm}$ symmetry of the pattern.

In the just described large-scale $4.8^{2}$ patterns, the principal manifestation of randomness, i.e., a variable orientation of the ornamental fill in the S-octagons, is frozen and tied to the $4.8^{2}$ grid. 'Liberation' of the T-octagons from mutual edge contacts in the move from the Lahore pattern to the Gwalior one, and the consequences of their rotations by $22.5^{\circ}$ into virtual vertex-contacts instead of edgecontacts, and of the filling of thus created additional areas by the S-octagons, led to the Gwalior pattern with a rotational freedom of the S-octagons and to a potentially disordered pattern.

Two other prominent jali screens are derivatives of the Lahore (or Jaipur) pattern. A prominent pattern (Fig. 13) from Salim Singh ki Haveli, built in 1815 in Jaisalmer

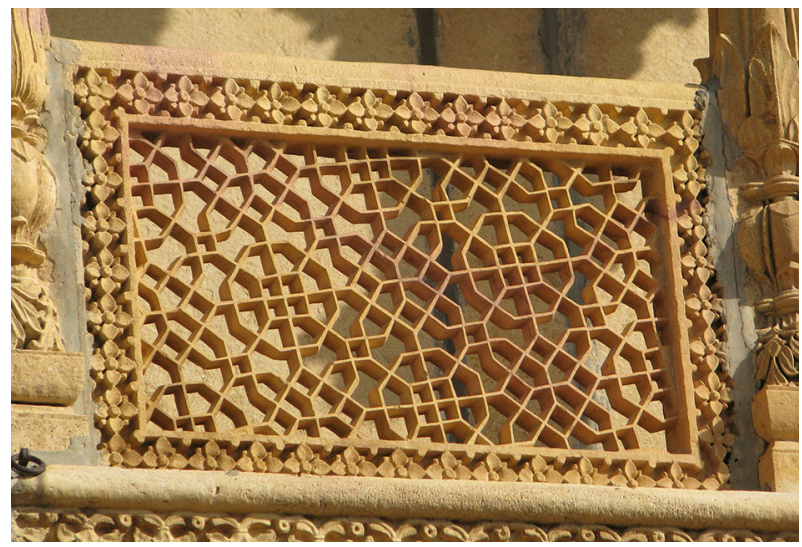

Fig. 13 A jali from Salim Singh ki Haveli, built in 1815 in Jaisalmer, an expanded form of the pattern from Fig. 11. Details in the text 
is obtained from the Lahore pattern when its square lattice parameters are extended by $a+a / \sqrt{ } 2$ so that formerly partly overlapping fourfold clusters of S-octagons became separated by this interval. This made insertion of the same motif shifted by $(1 / 2,1 / 2)$ possible and allowed use of symmetrically filled $4 \mathrm{~mm}$ octagons at $(0,1 / 2)$ and $(1 / 2,0)$, so that a centered mesh is obtained when the original axial directions are maintained. However, this pattern was copied from the Gwalior mausoleum complex. The pattern (Fig. 14) from Diwan-i-Khas of Fatehpur Sikri, built from 1571 to 1575 in Agra, Uttar Pradesh, is obtained from the previous one by extension of it by an additional $a \sqrt{ } 2$ segment. By this operation, the last partial overlap of the Gwalior-style S-octagons was removed and they contact one another via edges, forming an $8^{2} .4$ pattern of their own (Fig. 13). Both patterns are $p 4 m m$ and the star traceable in them is doubled in size.

It should be mentioned that the above treated patterns of parallel dumb-bells (Fig. 5) from a window of Humayun's Tomb (1557-1565, by Mirak Mirza Ghiyas) in Delhi represent a different lattice and symmetry, either $\mathrm{cmm}$ or its subgroups.

Let us mention a more distant but in some features similar 'next of kin': a hexagonal pattern from the Çifte Minare Medrese in Sivas, Turkey (built in 1271) (Fig. 15), visually reminiscent of the pattern from the Lahore Fort (Fig. 11). It contains edge-sharing $\mathrm{T}$-hexagons with a perimeter decorated by S-enneagons (which are shared by adjacent T-hexagons). The S-enneagons contain three 'Pbaguettes', each of which is shared with an adjacent S-enneagon, and three concave

Fig. 14 A jali from Diwan-iKhas of Fatehpur Sikri, built 1571-1575 in Agra, further expansion of the patterns from Figs. 11 and 12. Details in the text

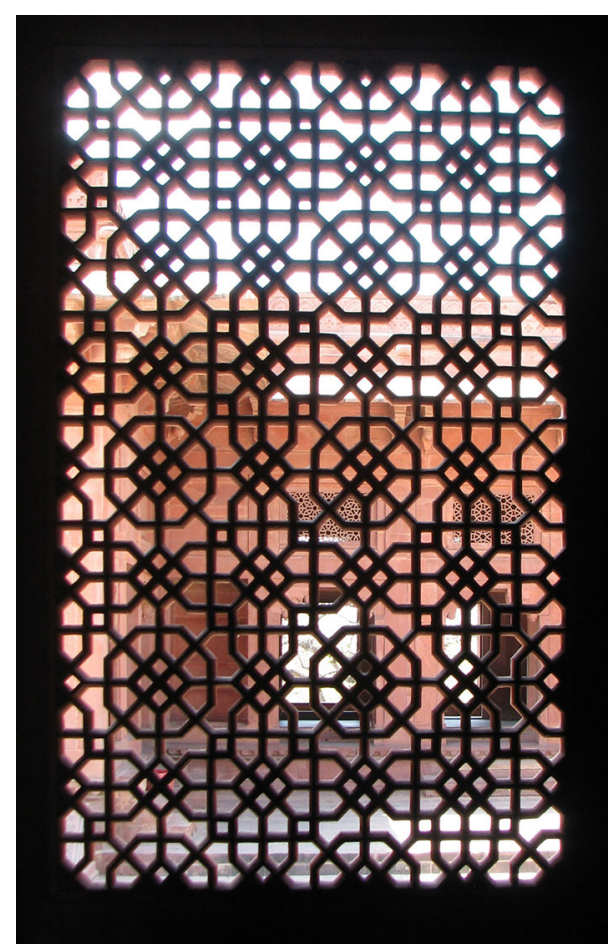


Fig. 15 A hexagonal pattern from the Çifte Minare Medrese in Sivas, Turkey (built in 1271 under an Ilkhanid governor) with T-hexagons and secondary, filled S-enneagons. Compare with the T-octagon-S-octagon combination from the Lahore Fort, shown in Fig. 11

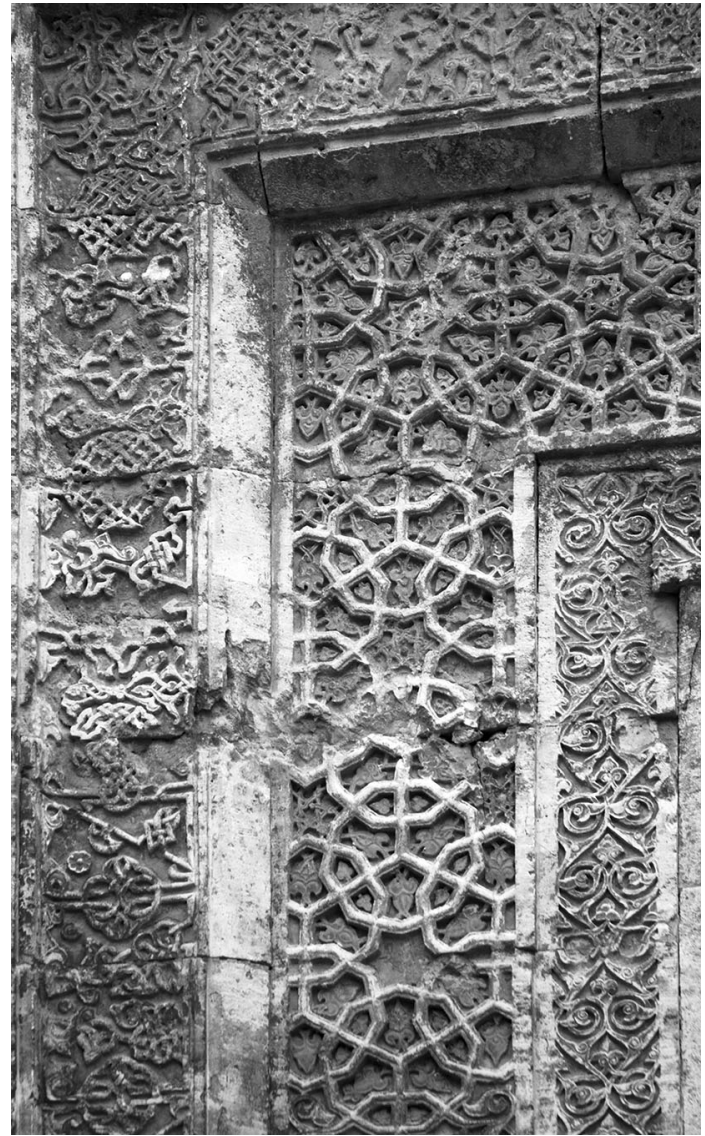

P-octagons (incomplete figures of eight). Thus, the $m$ symmetry of the Gwalior S-octagon is replaced by the $3 m$ symmetry of a filled enneagon. This pattern, which at a cursory look can be mistaken for the Gwalior pattern has been recently analyzed in detail by Redondo-Buitrago and Huylebrouck (2015). It is also present as a jali at the Agra Fort (built about 1550 by the Mughal dynasty), a balustrade in the Selimiye Camii in Edirne (1569-1575), Hagia Sophia and elsewhere in Istanbul (RedondoBuitrago and Huylebrouck 2015). It has also been copied at the Topkapi kiosks in Istanbul (1635-1639) but the above mentioned weather-beaten Sivas locality is a proof of its long tradition.

\section{Conclusions}

The studied jali screen from Gwalior is only one of several members of a regional pattern family which share the most typical pattern elements-the partly overlapping secondary octagons with asymmetric primary fill. We see a gradual development of the motifs, from symmetry-bound elements and patterns toward a 
high degree of rotational freedom. Both in Gwalior and Lahore these patterns are found side by side with strictly periodic highly-symmetrical 'static' patterns. At both localities they occur in the lower and easily accessible corners of the complex jali screen. Elsewhere, they form an entire openwork panel, especially when talking about the periodic pattern derivatives. Periodic patterns derived from the $4.8^{2}$ Lahore pattern form a family of extension and intercalation derivatives, showing the derivation methods of ancient artists for obtaining new patterns from old ones.

The complex fill of intermediate-size secondary octagons is a logical result of the geometry of the $4.8^{2}$ Lahore pattern. It was retained in the much more complex Gwalior pattern although it was freed from the original geometric constraints. Several alternative points of view and descriptions of the Gwalior pattern converge into a unified interpretation as a doubly-disordered pattern in which orientational disorder of elements generates positional disorder. A potential for a quasiperiodic character of patterns based on Gwalior-type tiles has been investigated in detail.

Acknowledgements Critical reading by Dr. J. Bailey and two anonymous referees contributed to the clarity of the final manuscript.

\section{References}

Broug, Eric. 2013. Islamic Geometric Design. New York: Thames \& Hudson.

Castéra, Jean-Marc. 2016. Persian variations. Nexus Network Journal, 18: 223-274.

Grünbaum, Branko and Shephard, G.C. 1987. Tilings and Patterns. New York: Freeman \& Co.

Janssen, T., Chapuis, G. and de Boissieu, M. 2007. Aperiodic Crystals. From Modulated Phases to Quasicrystals. Oxford: Oxford University Press.

Makovicky, Emil. 1992. 800-year-old pentagonal tiling from Maragha, Iran, and the new varieties of aperiodic tiling it inspired. In: Fivefold Symmetry, ed. Istvan Hargittai, 67-86. Singapore: World Scientific.

Makovicky, Emil. 2009. Another look at the Blue Tomb of Maragha, a site of the first quasicrystalline Islamic pattern. Symmetry: Culture and Science 19: 127-151.

Makovicky, Emil. 2015. In the footsteps of Maragha: ornamental panels in the madrasas and mosques of Esfahan, Konya, Agra, Sivas and Yazd. Symmetry: Culture and Science 26: 421-441.

Makovicky, Emil and Fenoll Hach-Ali, Purificacion. 1996. Mirador de Lindaraja: Islamic ornamental patterns based on quasi-periodic octagonal lattices in Alhambra, Granada, and Alcazar, Sevilla, Spain. Boletín de la Sociedad Española de Mineralogía 19: 1-26.

Makovicky, Emil and Fenoll Hach-Ali, Purificacion. 2001. The stalactite dome of the Sala de Dos Hermanas - an octagonal tiling? Boletín de la Sociedad Española de Mineralogía 24: 1-21.

Makovicky, Emil, Rull Pérez, Fernando and Fenoll Hach Alí, Purificacion. 1998. Decagonal patterns in the Islamic ornamental art of Spain and Morocco. Boletín de la Sociedad Española de Mineralogía 21: 107-127.

Porter, Yves and Degeorge, Gérard. 2009. The Glory of the Sultans. Islamic Architecture of India. Paris: Flammarion.

Redondo-Buitrago, A. and Huylebrouck, D. (2015) Nonagons in the Hagia Sophia and the Selimiye Mosque. Nexus Network Journal 17, 157-181.

Stierlin, Henri. 2002. Islamic Art and Architecture from Isfahan to the Taj Mahal. London: Thames \& Hudson.

Takeo Kamiya. 2004. The Guide to the Architecture of Indian Subcontinent. Goa: Architecture Autonomous.

Tillotson, Giles H.R. 1990. Mughal India. San Francisco: Chronicle Books. 
Emil Makovicky was born in Bratislava, Slovakia, in 1940. He obtained his M.Sc and RNDr. degrees at the University of Bratislava and Ph.D. from McGill University, Montreal, Canada (in 1970). After a stay at Yale University he obtained a stable position in mineralogy at the University of Copenhagen, Denmark in 1972 where he became professor in 1995, now as emeritus. His contributions to symmetry in art started in 1977 and include applications of diverse symmetry groups and quasiperiodicity by traditional artists and artisans.

Nicolette M. Makovicky was born in Gentofte, Denmark, in 1980. She obtained her M.Sc. from the University of Copenhagen and Ph.D. from the University College, London, UK. She holds a position in interdisciplinary area studies at the University of Oxford, UK. 